Coopérer avec la justice du roi. La peine des galères dans la République de Genève (1613-1788)

\title{
Robin Majeur
}

\section{(2) OpenEdition}

\section{Journals}

Édition électronique

URL : http://journals.openedition.org/chs/1592

DOI : $10.4000 /$ chs. 1592

ISSN : $1663-4837$

Éditeur

Librairie Droz

Édition imprimée

Date de publication : 1 novembre 2015

Pagination : $5-24$

ISBN : 978-2-600-01908-8

ISSN : 1422-0857

\section{Référence électronique}

Robin Majeur, "Coopérer avec la justice du roi. La peine des galères dans la République de Genève (1613-1788) », Crime, Histoire \& Sociétés / Crime, History \& Societies [En ligne], Vol. 19, n² | 2015, mis en ligne le 01 novembre 2017, consulté le 04 mai 2019. URL : http://journals.openedition.org/chs/1592 ; DOI : 10.4000/chs.1592 


\title{
Coopérer avec la justice du roi La peine des galères dans la République de Genève $(1613-1788)^{1}$
}

\author{
Robin Majeur ${ }^{2}$
}

Bien connue des historiens de la justice, la peine des galères a pourtant été peu étudiée sous l'angle de la coopération judiciaire. On trouve en effet, sur les bancs des galères et dans les bagnes, des hommes condamnés par des juridictions extérieures au territoire sur lequel ils purgent leur peine. Cet article se penche sur le cas de la République de Genève, qui a envoyé plus de cinquante criminels aux galères du roi de France entre 1613 et 1788. Loin de déposséder la République de son droit régalien de rendre la justice, la pratique des juges genevois contribue à renforcer les liens diplomatiques entre deux États au prestige inégal, en même temps qu'elle constitue une solution pénale efficace. Ces interrelations pragmatiques autour de la peine des galères, plutôt ponctuelles, marquées par des pratiques archä̈ques, forgent ainsi un socle commun sur lequel peuvent s'appuyer deux États souverains pour coopérer malgré leurs différences.

Penal servitude on the galleys is well known to historians of criminal justice, although sparsely researched from the perspective of cooperation between different judicial systems. Yet, on French galley benches and penal colonies (Bagnes), there were in fact men serving sentences which were issued by a court outside the French territorial jurisdiction. This article focuses on the case of the Genevan Republic, which set more than fifty convicted criminals to the French royal galleys between 1613 and 1788. Far from affecting the sovereign authority of the Genevan republic, the practice by Genevan judges of sending convicted criminals to French galleys helped to strengthen diplomatic ties between two states with very asymmetrical power relations, while at the same time providing an effective solution to a penal problem. These rather occasional, pragmatic arrangements surrounding penal servitude on the galleys, characterised by ancient practices, thus constituted a common pillar against which both sovereign states could lean and cooperate, despite their differences.

1 Cet article est tiré, sous une forme synthétique et actualisée, du mémoire de baccalauréat universitaire intitulé Genève et la pratique des condamnations aux galères (1613-1788), effectué sous la direction de Marco Cicchini et Michel Porret à la Faculté des lettres de 1’Université de Genève en 2009.

2 Robin Majeur a fait ses études à la Faculté des lettres de l'Université de Genève, en Suisse. D'abord intéressé par les problématiques liées à l'histoire sociale et institutionnelle de la justice, il a ensuite consacré son mémoire de maîtrise universitaire à la culture politique du procureur général genevois Jean-Robert Tronchin (1710-1793). Il travaille actuellement dans l'enseignement secondaire. 


\section{INTRODUCTION}

T a fascination exercée par les forçats de la mer et ultérieurement de la terre, Ltel Jean Valjean, a généré une abondante historiographie mettant en lumière les normes juridiques qui définissent la peine des galères, ses modalités d'exécution ou encore ses conséquences sociales sur les personnes qui la subissent. Les États méditerranéens ont été un terrain propice à des recherches sur la «mécanique concentrationnaire » (André Zysberg) des galères ${ }^{3}$. Les condamnés provenant d'une juridiction étrangère ont cependant suscité moins d'intérêt. Le sort de ces hommes, qui purgeaient une peine dans un État qui n'avait pas prononcé lui-même la sanction, était le résultat d'une coopération judiciaire transfrontalière. À ce propos, les cas des galériens bavarois transférés à Venise et des Suisses envoyés aux galères de France ont fait l'objet d'études ${ }^{4}$. Toutefois ces dernières, principalement fondées sur les sources en aval (émanant des galères et des bagnes) et non sur celles provenant des juridictions qui ont prononcé les peines en amont, n'éclairent pas totalement les enjeux de la coopération judiciaire entre des États à la taille et au prestige dissemblables. Plus récemment, Ludolf Pelizaeus a traité de la peine des galères et de la coopération judiciaire, mais essentiellement pour s'intéresser à la répression transfrontalière de la criminalité et non à la coopération spécifique qu'implique cette sanction pénale 5 .

L'histoire de la peine des galères est celle d'un double mouvement paradoxal. Son usage va en augmentant depuis le $\mathrm{XVI}^{\mathrm{e}}$ siècle alors que sa fonction originelle disparait rapidement : avec l'apparition des navires de haut bord, qui peuvent emporter une puissance de feu bien supérieure, les galères perdent beaucoup de leur intérêt militaire. Cela témoigne de la progressive autonomisation judiciaire de cette peine vis-à-vis des considérations politiques et militaires. Au milieu du XVIII ${ }^{e}$ siècle, en Espagne, en France, en Toscane, les galères, vétustes et délabrées, sont abandonnées. Les cours de justice continuent pourtant d'y envoyer des forçats, auxquels on trouve de nouvelles affectations. La peine des galères survit aux galères elles-mêmes. À témoin, ce glissement sémantique, observé par Angiolini dans plusieurs langues: les termes galère et galérien, attachés originellement aux navires à rames, font de plus en plus référence aux peines réclusives, que ce soit en français où galérien devient synonyme de forçat ou en italien et en espagnol où le terme galère se substitue tout bonnement à la prison ${ }^{6}$.

La République de Genève, sans accès à la mer et sans flotte de guerre, contribue à envoyer des hommes condamnés par la justice civile et militaire sur les galères d'États étrangers. Entre 1613 et 1788, les juges genevois prononcent 58 fois la peine des galères - un chiffre certes modeste au vu des quelque 18000 procédures criminelles engagées sous l'Ancien Régime, mais qui témoigne d'un besoin ponctuel de se débarrasser de certains criminels ${ }^{7}$. Les condamnés sont expédiés sur les galères du

Voir par exemple Lo Basso (2006) pour Venise; Angiolini (2006) pour la Toscane; Zysberg (1987) pour la France; Pike (1982) pour l'Espagne.

Schlosser (1984); Dumas (2005).

Pelizaeus (2011).

Angiolini (2006, pp. 93-94).

Ont été consultés pour cette étude tous les Registres du Conseil (dorénavant RC), séries 1-3, des Archives d'État de Genève (dorénavant AEG) portant sur l'Ancien Régime. 
roi de France. Qu'une petite République, réformée depuis 1536, délègue au roi Très Chrétien l'exécution d'une peine qu'elle a souverainement prononcée, ne manque pas d'interroger: n'est-ce pas se dessaisir de ses prérogatives judiciaires que de laisser le soin à d'autres juridictions d'exécuter la peine? N'est-ce pas renoncer au droit régalien de rendre la justice, au fondement de la souveraineté des États modernes? Plutôt que de voir un paradoxe dans cette pratique institutionnelle, on s'interrogera sur les modalités et sur les effets que produit la coopération judiciaire. Autrement dit, il s'agira de voir comment, dans les interrelations ponctuelles qu'engendrent les condamnations aux galères à Genève, se construit un socle commun qui permet à deux États de s'entendre au-delà de leurs différences confessionnelles, de forme de gouvernement, de prestige. Le cas genevois permettrait ainsi de mettre en lumière, malgré le nombre restreint de sentences aux galères, les aptitudes des États d'Ancien Régime à trouver des solutions pragmatiques et plutôt efficaces lorsqu'il s'agit de coopérer avec des voisins.

Dans le cas genevois, la peine des galères met en jeu une dimension spatiale: la République doit remettre ses condamnés aux officiers royaux à ses frontières. Cependant, à cette époque, l'État n'est pas encore conçu comme l'unité homogène, cohérente et bien délimitée qu'est l'État-nation. Au contraire, bien que Bodin ait étroitement articulé la notion de souveraineté - absolue, perpétuelle, indivisible et inaliénable - avec les États dès le $\mathrm{XVI}^{\mathrm{e}}$ siècle, la conception territoriale de l'État à l'époque moderne relève d'une grande complexité ${ }^{8}$. Le XVIII ${ }^{e}$ siècle genevois est d'ailleurs marqué par les tentatives de la République de régler ses contours territoriaux avec ses voisins à travers des «traités de limites»9. L'étude des condamnations aux galères permet donc de réfléchir non seulement à l'intégration d'une pénalité nouvelle dans l'appareil répressif d'une République, mais aussi aux effets produits par la territorialisation des pratiques en matière de définition de l'État. Dans son analyse de la genèse de l'État, Bourdieu rappelle que celui-ci «se fait en faisant $»^{10}$. À travers une approche anthropologique, centrée sur les acteurs et leurs pratiques, nous chercherons à voir comment le rituel judiciaire, dans sa dimension spatiale notamment, participe d'une affirmation du territoire souverain et contribue par-là même à renforcer l'État.

Nous présenterons successivement les deux logiques qui structurent l'usage de la peine des galères à Genève; celle, interne, qui est propre au fonctionnement d'un État souverain et celle, externe, qui relève de paramètres politiques et diplomatiques. Ces deux impulsions, tantôt concordantes, tantôt discordantes, expliquent sans doute à la fois l'existence et la rareté de cette peine à Genève. Nous nous pencherons, par la suite, sur la recrudescence des condamnations aux galères à la fin du XVIII ${ }^{e}$ siècle: comment se fait-il qu'une peine caractérisant si bien l'ancienne économie punitive puisse ressurgir au moment où les philosophes et réformateurs du droit commencent de renverser le paradigme du droit de punir? Il s'agira d'aller à rebours de toute tentation téléologique pour envisager la peine des galères non comme un archaïsme inéluctablement appelé à disparaître avec l'avènement de la modernité pénale, mais comme une possible réponse apportée par les juges genevois à une certaine forme de criminalité dans une période de mutation des normes juridiques.

\footnotetext{
8 Wüst, Müller (2011).

9 Rizzo (2003); Carpanetto (2007).

10 Bourdieu (2012, p. 198).
} 


\section{INSTITUTIONNALISATION D'UNE ROUTINE JUDICIAIRE}

Née au tournant de l'époque moderne, la peine des galères connaît sous l'Ancien Régime un usage massif dans l'espace européen. Les États méditerranéens, désireux de développer leur flotte de galères à des fins militaires et de prestige, trouvent avec cette peine une solution à la pénurie incessante de rameurs sur ces bâtiments gourmands en hommes. Aux esclaves qui peuplent les bancs des galères depuis l'Antiquité et aux volontaires (appelés bonevoglie) qui se font de plus en plus rares, s'ajoutent donc les forçats, condamnés par différentes juridictions aux galères à vie ou «à temps».

Il faut dire que la peine des galères s'intègre parfaitement à l'économie punitive d'Ancien Régime, qui répond à une logique expiatoire et volontiers éliminatoire, et s'articule autour de la publicité et de l'exemplarité de la peine. Ainsi, les galériens sont marqués dans leur chair par les lettres GAL inscrites derrière l'épaule; vêtus d'une casaque rouge, frappés d'infamie, ils subissent, en France par exemple, l'humiliation de traverser le pays avec l'une des trois chaînes qui sillonnent le royaume pour les conduire à Marseille, port d'attache principal des galères ${ }^{11}$. Là, comme sur les galères de tous les États européens, ils vivent l'expérience d'une promiscuité extrême, attachés à leur banc de rame sur un espace réduit. Au-delà de l'élimination sociale provoquée par l'infamie et l'éloignement, la peine des galères est souvent synonyme d'élimination physique: un condamné sur deux n'y survit $\operatorname{pas}^{12}$. Alliant souffrance corporelle, éloignement et enfermement, censée être d'une exemplarité éclatante par le rituel public qu'elle engendre, la peine des galères fait figure de condensé spectaculaire des modalités punitives d'Ancien Régime.

Les États possédant une marine de rame prospectent dès la fin du XVI ${ }^{\mathrm{e}}$ siècle auprès de juridictions étrangères. Plusieurs principautés allemandes et cantons suisses reçoivent ainsi des sollicitations de Gênes, Venise, la Savoie, l'Espagne et la France. C'est avec cette dernière que la Diète helvétique conclut un accord en 1601. Les Cantons disposent alors d'un moyen légal de livrer les détenus qui encombrent leurs prisons à l'ambassadeur de France à Soleure, en retour de quoi le roi s'engage à payer les frais des futurs galériens ${ }^{13}$. La République de Genève reçoit également des invitations d'États voisins. Un mémoire français daté du 11 mai 1613 appelle les autorités genevoises à «épargner la vie aux criminels qui ne seront atteints de crimes très atroces et [à] les condamner à résider punis aux galères du roi ». De là sans doute la première condamnation aux galères genevoise durant cette même année. Mais l'embarras des autorités «pour n'avoir encore été tel supplice pratiqué» les conduit à commuer la peine initiale. On propose au condamné l'office de bourreau, qu'il accepte. Cette première tentative avortée révèle les difficultés d'application d'une peine dont l'exécution n'est pas du ressort de l'autorité judiciaire qui la prononce: malgré la volonté affichée par les autorités françaises d'accueillir des galériens, les soubassements d'une coopération judiciaire entre Genève et la France ne semblent pas être en place pour permettre le bon déroulement de l'opération.

La peine des galères s'institutionnalise à Genève dans la moitié des années 1680. La politique de puissance et de gloire du roi Louis XIV y est pour beaucoup.

11 Rappaport (2006, pp. 11-17).

12 Zysberg (1987, pp. 347-350).

13 Dumas (2005, pp. 57-68). 
Sous l'impulsion de son ministre Colbert, d'importantes réformes sont entreprises pour renforcer le corps des galères: embauche de bonevoglie, injonctions faites aux juridictions du royaume d'infliger la peine des galères, rationalisation des itinéraires des chaînes de forçats pour quadriller le territoire. L'ordonnance du 4 décembre 1684 substitue la peine des galères à la peine de mort pour les déserteurs. Une année plus tard, l'édit de Fontainebleau permet d'envoyer aux galères les protestants tentant de fuir le pays. C'est donc au moment de ce grand appel d'air du côté du royaume de France que les juges genevois s'attellent à prononcer la peine des galères. Ils y reçoivent des encouragements indirects à travers l'accueil très favorable que font les autorités françaises à l'annonce d'un condamné ${ }^{14}$. Entre 1685 et 1715, ils prononcent la peine à 24 reprises au moins, soit près de la moitié du nombre total de condamnations aux galères genevoises.

Sous l'Ancien Régime, les sources juridiques qui permettent la qualification du crime et la motivation de la peine sont éparses. À Genève, les juges du Petit Conseil - dont la sentence est prononcée en dernier ressort - s'appuient sur quatre différents types de sources: le droit romain, les lois promulguées au cours du temps, la doctrine et enfin la jurisprudence genevoise et étrangère. La peine des galères n'apparaît pas dans les édits criminels de la République. Elle existe en revanche, dans les pays voisins, depuis le $\mathrm{XVI}^{\mathrm{e}}$ siècle. Sur l'échelle des peines, elle figure très haut: en France, l'Ordonnance criminelle de 1670 place les galères perpétuelles en troisième position, juste derrière la mort et la question avec réserve de preuve ${ }^{15}$. Contrairement au bannissement à perpétuité, c'est une peine qui ne laisse aucun droit au condamné. Elle peut cependant être déclinée de différentes façons lorsqu'elle est prononcée «à temps». Dans ces cas, elle se situe plus bas sur l'échelle des peines puisqu'elle n'entraîne pas la mort civile, ni ne dépossède le galérien de tous ses biens. Celui-ci peut théoriquement recouvrer la liberté et se réinsérer socialement au terme de sa peine, bien qu'il soit assigné à son dernier domicile et porteur de l'infamante marque «GAL».

Les crimes sanctionnés par cette peine sévère dans les cours européennes s'étendent des crimes de sang à la désertion en passant par la contrebande. Louis XIV y ajoute les huguenots qui tentent de quitter le royaume dès 1685 , avec la Révocation de l'Édit de Nantes. À Genève, où des efforts sont faits pour porter secours aux condamnés «pour la foi $»^{16}$, prédomine la répression des crimes contre les biens. Avec $38 \%$, ils arrivent en tête devant les délits de désertion. Viennent ensuite les crimes de sang, la contrefaçon et la rupture de ban. Plus rarement, des cas de diffamation, mendicité ou vagabondage conduisent les juges genevois à prononcer la peine des galères. Le spectre des délits réprimés par cette peine est très large et l'historien peine à y déceler une cohérence; d'autant plus que les crimes contre les biens, majoritaires, peuvent aller du vol qualifié, nocturne et prémédité, au petit larcin commis sur la place publique un jour de marché. Il faut toutefois être attentif à un élément. Si l'on prend en considération non la durée des peines, mais seulement la distinction entre galères «à temps » et galères à perpétuité, on s'aperçoit que celles-ci viennent sanctionner une majorité de crimes de sang quand celles-là punissent principalement des crimes contre les biens.

\footnotetext{
14 AEG RC 187, 28 mai 1687, p. 111.

15 Serpillon (1777, pp. 1077-1082).

16 Grandjean (1981).
} 
Si le contentieux apparaît vaste et peu défini, la catégorie de population visée par la peine des galères est assez caractérisée. En premier lieu, il ne s'agit que d'hommes. Selon la doctrine, «la décence de leur sexe» impose aux femmes un autre type de peine. Elles sont généralement condamnées à la détention en maison de force ${ }^{17}$; une philosophie pénale que rejoignent des considérations bien pragmatiques: les hommes de chiourme recherchés doivent être extrêmement robustes pour supporter le travail de la vogue (jusqu'au début du XVIII ${ }^{e}$ siècle) et les travaux pénibles dans les arsenaux ou dans les bagnes. Pour cette même raison, l'âge des condamnés est relativement homogène: la moyenne s'établit à 27 ans. Mieux vaut des hommes en pleine force de l'âge et disposant de tous leurs moyens physiques. Il arrive ainsi que les juges genevois renoncent à mettre à exécution une sentence de galères suite à la dégradation physique d'un condamné ${ }^{18}$. Ils n'hésitent pas à commuer les 10 ans de galères de Jean-Pierre Dumas, alors que celui-ci a défié leur autorité en tentant de s'échapper de prison avant d'être remis aux officiers français:

Le Conseil faisant réflexion sur le rapport fait céans par noble Jean-Robert Chouet de l'accident arrivé audit Dumas, qui s'est rompu et brisé la jambe ces jours passés en voulant se sauver des prisons après avoir rompu les fers qu'il avait aux pieds et aux mains, et iceux ouverts, on l'a libéré eu égard à cet accident par lequel il a beaucoup souffert et n'était plus capable ni propre au service des galères, demeurant cependant au bannissement perpétuel à peine de la vie ${ }^{19}$.

Joue vraisemblablement dans l'esprit des juges la menace d'une opposition des officiers royaux. Ceux-ci peuvent en effet refuser un condamné en fonction d'un physique jugé inadapté à la condition de galérien ${ }^{20}$. Le coup est pénible à encaisser pour la République puisque les frais de prison, où patientent les condamnés, sont à sa charge et qu'elle ne retire aucune récompense, ne serait-ce que symbolique, d'avoir prononcé une sentence de galères inutile au roi de France. L'intérêt de l'État veut donc qu'on minimise ce genre de risques en concentrant cette peine sur des justiciables à la condition physique irréprochable.

Exception faite de la fin du XVIII ${ }^{e}$ siècle, les condamnés aux galères se distinguent par leur provenance géographique uniforme : jusqu'en 1738, les étrangers représentent $84 \%$ de la population frappée d'une peine de galères à Genève. Des quatre Genevois condamnés durant cette période, trois en réchappent grâce à des commutations de peine. Les étrangers proviennent en grande majorité de France, mais aussi de Savoie, du Piémont et même de Hongrie. Arrivés en ville depuis peu pour de prétendues raisons de commerce, ils disent loger dans des auberges locales. Pour cette catégorie de population peu intégrée au tissu social genevois ${ }^{21}$, une pénalité d'élimination semble facilement acceptable. Au contraire du bannissement dont le contrôle est limité, la peine des galères permet une plus grande efficacité dans son application: emmenés à des centaines de kilomètres de la République, enchaînés et surveillés, les galériens sont moins susceptibles de revenir semer le trouble à

Muyart de Vouglans (1780, p. 62).

18 AEG RC 188, 24 mars 1688, p. 129. Les peines de galères sont généralement commuées en bannissement à perpétuité.

19 AEG RC 188, 3 mars 1688, pp. 95-96.

20 AEG Procès Criminel (dorénavant PC) 4875, 1691.

${ }^{21}$ Il convient toutefois de nuancer cette affirmation en précisant que plusieurs de ces étrangers proviennent de régions limitrophes à Genève. 
Genève. Tout se passe donc comme si la peine des galères, certes intégrée à l'arsenal pénal genevois, ne fait l'objet que d'une utilisation très sélective.

Entre une sentence de galères et la remise effective du condamné aux autorités françaises, il existe une marge importante que révèle bien une statistique: seuls 58\% des condamnés genevois purgent leur peine. Plusieurs éléments expliquent ce qui semble être une inefficacité judiciaire crasse. Les juges condamnent parfois aux galères par contumace. Preuve supplémentaire, mais aussi circonstance aggravante, la contumace entraîne volontiers la sévérité des juges. Leur verdict, même s'il a peu de chances d'envoyer réellement le condamné aux galères, leur ménage la possibilité d'une rigueur maximale en cas de récidive. Les commutations de peine contribuent à faire baisser la statistique. Si les juges du Petit Conseil statuent en dernier ressort, le condamné a la possibilité, dans certains cas, de recourir au Conseil des Deux Cents, qui dispose du droit de grâce. Il peut donc voir sa sanction mitigée. D'autre part, les juges du Petit Conseil eux-mêmes sont parfois obligés de revenir sur leur décision quand le condamné présente des signes d'une santé défaillante ou que se manifestent des difficultés de collaboration avec les autorités françaises. Enfin, les cas d'évasions révèlent le problème de la porosité des prisons d'Ancien Régime, que la vétusté, le système de garde et le va-et-vient entre intérieur et extérieur rendent peu efficaces. Mais le pourcentage relativement peu élevé de condamnations aux galères purgées ne doit pas cacher, en définitive, l'intérêt que trouvent les autorités genevoises à cette peine, comme le prouve le simple fait qu'elles y ont recours sans être expressément tenues de le faire.

\section{LES PARAMÈTRES POLITIQUES ET DIPLOMATIQUES DE LA PEINE DES GALÈRES}

Si la peine des galères a un intérêt judiciaire pour la République de Genève, notamment en matière de sécurité intérieure, elle est cependant étroitement dépendante de la volonté politique du puissant voisin français. En témoigne la rapide décrue des condamnations après la mort de Louis XIV en 1715, quand toute idée de maintenir une forte flotte de galères à des fins de grandeur est abandonnée. Ce changement de cap politique devient problématique pour la machine judiciaire. La peine des galères est tout à fait institutionnalisée et continue d'être prononcée. À Genève, on le fait encore à cinq reprises entre 1722 et 1738, malgré le désintérêt des autorités françaises. Comme le montre Zysberg, le paradigme s'est inversé: avant 1715 , on utilise la peine des galères pour satisfaire le besoin de renforcer le corps des galères; après, on maintient celui-ci pour satisfaire l'exécution des condamnations aux galères infligées régulièrement par les juridictions du royaume ${ }^{22}$. Celles qui sont hors du pays éprouvent plus de difficultés. Ainsi, le manque de volonté français exaspère les autorités genevoises qui s'évertuent à chercher la collaboration de leur voisin pour livrer leurs condamnés. Elles font parvenir un mémoire au ministre des Affaires étrangères par l'intermédiaire de leur représentation diplomatique à Paris $^{23}$. La République ne peut se permettre d'attendre indéfiniment une réponse française. En 1730, on décide par exemple de commuer la peine d'André Romano

\footnotetext{
22 Zysberg (1987, p. 378).

23 AEG RC 238, 1738, pp. 60, 64, 357.
} 
en un bannissement à perpétuité «puisque les officiers de France n'ont pas voulu s'en charger jusqu'à présent, quelque diligence que l'on ait faite» ${ }^{24}$. Le désintérêt français a raison de la volonté genevoise puisqu'aucune condamnation aux galères n'est plus prononcée entre 1738 et $1781^{25}$.

Pour expliquer le monopole de la France comme lieu de réception des condamnés aux galères genevois, il faut souligner le rôle important du résident de France à Genève $^{26}$. Installé dans la République depuis 1679 , ce diplomate de bas rang s'occupe des affaires courantes touchant au rapport entre les autorités genevoises et la Cour du roi. Dès que les problèmes sont trop sérieux, en revanche, notamment lors des troubles politiques internes qui secouent la République au cours du XVIII ${ }^{e}$ siècle, il s'efface au profit d'un ministre plénipotentiaire venu directement de France ${ }^{27}$. Les condamnations aux galères, malgré leur parcimonie, font partie des affaires courantes. Aussitôt qu'ils ont prononcé une peine de galères, les juges genevois préviennent le résident, à charge pour lui de transmettre l'information à la Cour. Suivant les ordres qu'il en reçoit, il peut s'affairer aux aspects logistiques de l'opération: confirmation aux autorités genevoises de l'approbation française, contact avec les officiers de la maréchaussée de Gex, arrêt d'une date pour la remise du condamné. Bien que la République passe parfois directement par le canal de son représentant à Versailles, le résident est, dans la grande majorité des cas, l'interlocuteur privilégié. Sa présence joue comme une garantie, pour les autorités genevoises, que la délicate opération de délégation punitive pourra se passer correctement; du moins qu'un dialogue constant sera entretenu avec le puissant royaume voisin. Et cette assurance convainc pour un temps les autorités genevoises qu'avec un tel relais diplomatique, elles pourront user de la peine des galères sans risquer d'être confrontées à ce qu'elles craignent le plus: une attente indéfinie du condamné en prison, provoquée par un marasme diplomatique, et aux frais de la République.

Une lecture des sources en négatif permet d'accréditer l'idée du rôle prépondérant du résident de France dans l'usage de la peine des galères à Genève. Ainsi, dans les deux cas où les juges genevois prononcent une sentence de galères destinée à être exécutée ailleurs qu'en France, la peine n'est finalement pas appliquée. En 1669, ils condamnent un dénommé Louis Jordan aux galères vénitiennes. Mais la sollicitation du lointain relais diplomatique que constitue le représentant vénitien à Zurich s'avère compliquée et ne débouche sur rien. Jordan est banni ${ }^{28}$. De la même manière, deux condamnations aux galères en 1770 sont prononcées dans l'espoir d'envoyer les criminels sur les galères de Sardaigne. Des membres du gouvernement genevois tentent d'activer certains contacts dans le royaume voisin, sans succès. Les deux galériens voient leur peine commuée en bannissement à perpétuitée ${ }^{2}$. Dans ces cas, il semble qu'il manque un relais diplomatique institutionnalisé pour faire

AEG RC 232, 9 février 1732, p. 59.

25 Il existe une exception: trois peines de galères sont prononcées en 1770, avec l'espoir de remettre les condamnés aux autorités du royaume de Piémont-Sardaigne. Mais les contacts diplomatiques ne sont pas fructueux et la peine est finalement commuée en bannissement à perpétuité. Voir AEG PC 11942, $1769-1770$.

26 Jamais, à notre connaissance, un individu condamné aux galères à Genève n'a été envoyé ailleurs que sur les galères du roi de France.

27 Brandli (2006, pp. 77-168).

28 AEG PC 4037, 1669 et AEG PC 4196, 1672.

29 AEG PC 11942, 1770. 
œuvre de courroie de transmission. Plaque tournante sans grand pouvoir de décision, le résident de France est bien la cheville ouvrière de la coopération judiciaire que nécessite la pratique des condamnations aux galères à Genève. En ce sens, son établissement dès 1679 contribue grandement à faire expédier les galériens genevois sur sol français.

Deux étapes de la procédure illustrent la délicatesse de la coopération judiciaire: l'attente des condamnés en prison et leur remise aux autorités françaises. Après sa condamnation, le prévenu est en effet placé en prison jusqu'à ce qu'une date soit arrêtée pour effectuer sa remise aux officiers royaux. Dans toutes les procédures recensées entre 1685 et 1715, l'attente dure environ un mois. Un temps relativement court si l'on compte les détails à régler: contact avec le résident de France, attente de l'accord de Versailles, arrêt d'une date pour la remise, mise au propre de la sentence et d'une éventuelle fiche de signalement du condamné. C'est dire que la coopération judiciaire, loin d'être toujours fastidieuse, se passe généralement bien. Durant cette période, plusieurs conditions nécessaires à la bonne marche de ce type d'opérations sont réunies: l'habitude nouvelle des juges genevois à prononcer la peine des galères, la forte demande en galériens de la France de Louis XIV et une certaine routine dans la pratique des remises de condamnés. La bonne volonté de la France est primordiale. Après la mort de Louis XIV, la République de Genève peine à se débarrasser de ses condamnés aux galères. Le temps d'attente dans les prisons augmente: il est d'environ 3 mois en 1728, de 7 mois en 1738, avant l'abandon de cette pratique judiciaire jusqu'aux années $1780^{30}$. Le problème est essentiellement économique. La République assume les frais de prison jusqu'à la prononciation du jugement. Une fois celui-ci rendu, ils sont mis sur le compte de la France ${ }^{31}$. Mais l'attente prolongée d'un condamné aux galères donne à craindre que l'opération ne soit pas menée à son terme, d'où une certaine impatience genevoise lorsque cela ne se passe pas comme prévu.

La remise des condamnés constitue l'aboutissement de la procédure. Elle marque la fin de l'autorité de la République sur ses condamnés en la transférant aux officiers du roi de France. Moment délicat puisqu'il met en jeu la souveraineté des deux États qui coopèrent, il fait l'objet d'un déroulement ritualisé et marqué au sceau de la solennité. À la date convenue, les deux délégations se rendent sur la plaine de Châtelaine, située à la frontière. La délégation française est généralement menée par un officier de la maréchaussée du bailliage de Gex, accompagné de greffiers et de quelques hommes d'armes. Côté genevois, la délégation a toujours un conseiller à sa tête pendant la période louis-quatorzienne ${ }^{32}$. Il s'agit souvent du conseiller commis aux prisons, mais il peut arriver que le conseiller soit spécialement commis pour la tâche. Il est accompagné du géôlier et d'une escorte militaire. La présence d'un membre du Petit Conseil témoigne de l'importance de la délégation et surtout de cette culture politique d'Ancien Régime encore fortement basée sur la présence effective des acteurs. À l'inverse, à la fin du XVIII' siècle les conseillers ne se déplacent plus,

\footnotetext{
30 AEG PC 7570, 1728; AEG PC 7574, 1728; AEG PC 8574, 1738.

31 AEG Registre d'Écrou (dorénavant RE) Jur. Pen. H4.5, 1685, p. 93; AEG RC 228, 4 février 1729, p. 55.

32 Un conseiller est un membre du Petit Conseil, l'organe politique et judiciaire suprême de la République.
} 
signe peut-être de l'évolution du répertoire symbolique qui entoure cette pratique désormais dépourvue de tout éclat diplomatique.

Sur place, les deux délégations se positionnent à l'extrême limite de leur territoire respectif, sans jamais l'outrepasser. Les procès-verbaux mentionnent avec précision l'emplacement de chacun lors de la remise:

En exécution de ma commission, je me suis exprès transporté aujourd'hui aux limites des terres de la République, accompagné du sieur Porran, concierge des prisons de cette ville, d'un caporal et de quatre grenadiers conduisant le susdit Joseph Bonnard, où nous avons trouvé le sieur Jacquemet, brigadier de la maréchaussée de Gex, lequel m'a justifié être venu exprès pour recevoir ledit prisonnier que je lui ai remis, lui étant sur terre de France et moi sur celle de la République ${ }^{33}$.

Une fois les échanges effectués, il reste au deux têtes de délégation à dresser un procès-verbal de la remise. Très formel, il rend un compte détaillé de l'affaire. Il peut être rédigé par l'une ou l'autre des parties puis cosigné ${ }^{34}$ ou donner lieu à deux rédactions disjointes ${ }^{35}$. Le tout est fait à double pour que chacun en emporte une version. Les délégations se séparent alors, l'une revenant vers la cité genevoise, l'autre se dirigeant vers Lyon ou Besançon, villes où passe la chaîne des forçats. De là, les galériens sont emmenés à Marseille jusqu'au début du XVIII siècle, et aux bagnes de Toulon ou de Brest dans les années 1780. Ainsi, par sa codification, manifestée dans des procès-verbaux stéréotypés, la remise des condamnés, en définissant solennellement la dimension spatiale de chaque État, permet une reconnaissance mutuelle forte, qui marque et renforce la souveraineté de la petite République de Genève.

La délégation punitive est une pratique judiciaire qui ne se confond donc pas avec une allégeance. Elle permet à la petite République de traiter avec le royaume de France, non d'égal à égal, mais comme interlocuteur reconnu et considéré36. C'est aussi une manière, à une époque où elle n'a pas encore défini tous les contours diplomatiques de son rapport avec la France, de se montrer comme un partenaire stable et bien intentionné. Mais le versant politique de cette pratique la rend fragile. L'État genevois, qui inflige la peine des galères, n'est pas maître de son application et donc de son aboutissement. Il n'a que très peu de moyens de s'assurer de l'exacte exécution de ses sentences, notamment de celles qui sont prononcées «à temps». Lors de la période louis-quatorzienne, la durée des peines n'est pas respectée. Le roi ne choisit de se séparer des éléments de sa chiourme qu'au moment où il n'en a plus besoin. Partant, il est difficile de se faire entendre pour les juges de la petite République de Genève - tout comme pour les juridictions du royaume de France qui réclament leurs condamnés d'ailleurs ${ }^{37}$. Leur seul recours est la voie diplomatique, sous la forme d'une demande adressée au résident de France. Celle-ci ne se fait que

33 AEG PC 15443, 1788, «Rapport du sieur adjudant Desplances sur l'extradition de Joseph Bonnard du 31 août 1788 ».

34 AEG PC 4659, 1685, «Verbal».

35 Parmi d'autres AEG PC 4707, 1686, «Verbal»; AEG PC 4772, 1688, «Verbal»; AEG PC 5021, 1694, «Verbal».

36 Sur le rapport diplomatique complexe, ni égalitaire ni subordonné, entre une petite République et une grande monarchie, voir Brandli (2012).

37 Zysberg (1987, p. 365). 
sur requête du galérien lui-même, qui, selon l'usage, fait parvenir sa supplique à la cour de justice qui l'a condamné. Parfois y est joint un présent pour s'attirer la faveur des autorités. Ces dernières examinent la requête rapidement. Une pratique assez courante, si l'on estime que le condamné a légitimement le droit de quitter les galères, est de proposer un échange avec un autre criminel ${ }^{38}$. Aucune source ne permet cependant de dire que cela ait jamais réussi. On se contente généralement d'envoyer au requérant un extrait de son jugement et de parler du cas au résident de France. En fait, les autorités genevoises ne semblent pas tenir coûte que coûte à l'application exacte de leur sentence. Conscientes de leur faible marge de manœuvre, elles n'entrent que peu en matière sur de telles requêtes pour éviter, peut-être, de révéler leur impuissance.

\section{LES GALÈRES ET LA MODERNITÉ PÉNALE}

Le 20 octobre 1782, la République de Genève et le royaume de France signent une convention pour la remise à la France des condamnés aux galères du ressort juridique genevois. Alors que les juges genevois n'utilisent quasiment plus cette peine depuis 1738, ils rendent 22 condamnations aux galères entre 1781 et 1788 , soit plus d'un tiers du nombre total des condamnations aux galères prononcées depuis 1613. L'accord conclu en 1782 est l'aboutissement de quatre années de tractations diplomatiques entamées sous l'impulsion de la République. Il jette des bases stables pour les transferts de galériens, principalement à propos des questions financières. La convention prévoit le paiement de 180 florins par la République pour chaque galérien remis aux autorités françaises, cette somme couvrant les frais de transport jusqu'à Lyon ou Besançon, ainsi que la nourriture et l'attente dans les prisons jusqu'au passage de la chaîne ${ }^{39}$. Aussi, à la fin du XVIII ${ }^{\mathrm{e}}$ siècle, les termes de la coopération judiciaire entre Genève et la France en matière de galériens se sont-ils renversés: la France ne paie plus, elle encaisse; elle ne réclame plus, elle concède. Lorsque Sartine, secrétaire d'État à la Marine, est informé de la volonté genevoise d'expédier des condamnés sur les galères du roi, il déclare que «Sa Majesté veut bien consentir qu'ils y soient reçus $»^{40}$. Pour comprendre la résurrection de la peine des galères par les autorités genevoises, il faut s'intéresser à l'évolution des discours et des pratiques autour de cette peine au XVIII' siècle.

Avec son petit Traité des délits et des peines, publié anonymement en 1764, le marquis de Beccaria critique sévèrement la justice criminelle de son temps, fondée sur le spectacle de la souffrance physique. L'ouvrage propose un nouveau paradigme pénal. Beccaria cherche à séparer définitivement la sphère pénale de la sphère religieuse : un délit n'est plus un péché, mais une infraction sociale. La sécularisation du contentieux criminel entraîne la sécularisation de la sanction. Purger sa peine n'est plus expier sa faute, mais réparer le tort causé à la société. Beccaria inscrit sa pensée dans la fiction du contrat social. Un criminel est un individu qui a rompu le pacte social. Pour réparer le tort causé à la collectivité et pour réintégrer le corps

\footnotetext{
$38 \quad$ AEG RC 196, p. 104.

39 AEG RC 283, 21 octobre 1782, annexe p. 415.

40 AEG RC 279, $1^{\text {er }}$ octobre 1778, «Copie de la lettre de Monsieur de Sartine à Monsieur le comte de Vergennes ».
} 
social, le criminel doit accomplir une peine dont la société dans son ensemble est bénéficiaire. L'économie punitive éliminatoire se voit remplacée par une économie punitive corrective: la justice doit avoir pour fin non l'élimination du criminel, mais la resocialisation. À côté de cet idéal correctif se lit une pensée empreinte d'utilitarisme. Le coût humain de la justice doit être minimal et la peine contribuer au bonheur du plus grand nombre.

Selon le mot de Foucault, l'idée de Beccaria et des réformateurs n'est pas de punir moins, mais de punir mieux ${ }^{41}$. La sévérité des châtiments conduit souvent à l'impunité. Les juges appliquent rarement les peines extrêmes qu'imposent les lois. Les justiciables, d'autre part, hésitent à recourir en justice sachant par quelle sévérité on peut punir les plus petits délits. De plus, le spectacle du châtiment sévère n'a jamais prouvé son efficacité préventive. À tout cela s'ajoute une exigence nouvelle d'humanité, qui invite à modérer les peines. Mieux que l'éclat des supplices, la certitude de la peine saura dissuader les hommes. La peine doit être modérée, strictement nécessaire et proportionnée au délit. La sévérité d'une peine s'appréciera moins à l'intensité de la souffrance infligée qu'à sa durée. Pour Beccaria, la prévention du crime passe par une meilleure lisibilité de la justice criminelle. Le rapport entre crime et peine doit être limpide. L'arbitraire des juges, si important dans l'Ancien Régime pénal, n'a plus sa place. Il faut des lois fixant clairement les peines à appliquer proportionnellement à chaque délit. L'idée d'une codification des lois est en gestation ${ }^{42}$.

La peine des galères est-elle compatible avec les idées des réformateurs? Elle caractérise si bien l'ancienne économie pénale qu'on l'imagine a priori mal trouver grâce à leurs yeux. En fait, moyennant un certain nombre d'aménagements, la peine des galères s'assure généralement une bonne place dans leurs systèmes. Ce qui retient essentiellement leur intérêt est le «double principe organique de la peine des galères, c'est-à-dire priver le condamné de sa liberté et mettre sa puissance de travail au service d'un intérêt supérieur $\gg^{43}$.

La sortie du régime suppliciaire impose de faire souffrir moins physiquement que moralement. Les maux endurés dans leur chair par les forçats doivent être réduits au minimum. Ce n'est plus le corps qu'il faut punir, mais l'âme. L'évolution de la conception de la liberté, passée progressivement d'une définition plurielle fondée sur les privilèges d'une société scindée en «ordres » à une définition singulière attachée aux droits de l'individu, permet d'envisager une nouvelle tactique punitive: la privation de la liberté. Devenue un bien précieux pour chaque individu, la liberté est un levier sur lequel peut s'appuyer le nouveau régime pénal. Et la peine des galères, grâce à sa dimension carcérale, est en mesure d'y jouer un rôle. Le dommage moral subi par le forçat contribuera non seulement à lui faire payer son crime, mais aussi à lui faire sentir l'avantage de ne pas transgresser à nouveau les lois :

L'homme privé de sa liberté apprend à la chérir encore davantage, et son propre intérêt qui se développe à ses yeux dans ses vrais rapports avec les intérêts des autres lui fait une loi sentie de se conformer à l'ordre établi quand il aura recouvré sa liberté ${ }^{44}$.

\footnotetext{
Foucault (1975, pp. 96-98).

42 Porret (2003).

43. Vigié (1988, p. 413).

44 Dufriche de Valazé (1784, p. 299).
} 
Des multiples dimensions punitives de la peine des galères, on retient celle, essentielle, de la privation de la liberté qui garantit à la société une sécurité maximale tout en permettant de satisfaire aux finalités nouvelles du droit de punir: être utile à la société et au délinquant. Dans l'Encyclopédie déjà, l'argument utilitaire est mis en avant: la peine des galères, vue comme une sanction atténuée de la peine capitale et «plus conforme aux lois de l'humanité», a l'avantage de conserver à l'Etat des hommes dont il pourra profiter ${ }^{45}$. Les réformateurs, bien loin d'être tous abolitionnistes, soulignent «l'inutilité» de la peine de mort en comparaison des peines qui préservent l'intégrité physique des condamnés: «en disposant de sa vie, on prive la société d'un citoyen qui lui serait utile, mais il continue à l'être dans les fers. Le captif la sert encore dans ses travaux, l'homme mort est perdu pour elle ${ }^{46}$. Employée à des travaux publics et non plus uniquement à la vogue sur les galères, la force de travail des galériens est une valeur qui s'ajoute à la finalité sécuritaire de la philosophie pénale. Utile, le labeur du forçat a également une valeur corrective. Il permet d'éviter autant l'oisiveté que l'état de nécessité, deux facteurs criminogènes. Le désœuvrement est en effet vu comme le moyen offert à un individu livré à luimême de tomber dans la délinquance. D'autre part, tenant compte de cette équation sociale dangereuse qui fait résulter le crime de la nécessité, on vise à empêcher le système punitif de produire des indigents ${ }^{47}$. Le travail durant la peine constitue donc un atout après celle-ci puisque le forçat repenti, aguerri aux activités manuelles, aura un moyen de se réinsérer dans la société. Il est une ressource essentielle contre la récidive des galériens:

On doit avoir attention de les occuper pendant qu'on les tient dans l'esclavage à des travaux qu'ils puissent continuer lorsqu'ils auront recouvré la liberté. Autrement, ils ne seront pas meilleurs qu'auparavant, et c'est là une des raisons pour lesquelles ceux qui sortent des galères viennent pour l'ordinaire périr sur l'échafaud ${ }^{48}$.

L'utilité de la peine est consubstantielle à son pouvoir correctif: sans ce dernier, la société ne gagne rien à voir un individu la réintégrer. Mais la finalité corrective, en plus du travail des condamnés, passe par d'autres mesures importantes. Dans l'addition à l'entrée "galères» de l'Encyclopédie méthodique, Jacques-Vincent Delacroix (1743-1832), avocat au Parlement de Paris, dénonce l'école du crime qu'est le bagne ${ }^{49}$. Un des problèmes de la peine des galères est l'amplitude du contentieux qu'elle sanctionne. Des crimes aussi différents que le faux-saunage, le vol, l'homicide ou la contrefaçon peuvent être punis par la même peine. Et sa durée varie tellement selon les cas qu'elle semble relever de l'arbitraire plus que d'une juste mesure ${ }^{50}$. Mettant en contact les petits délinquants avec les pires criminels, pour un temps qui paraît aléatoire, le bagne, loin de moraliser ou de corriger, endurcit les

\footnotetext{
45 Encyclopédie $(1757$, t. 7, p. 445).

46 Pastoret (1770, t. 1, p. 32).

47 Encyclopédie méthodique, Jurisprudence (1784, t. 4, p. 693).

48 Brissot de Warville (1781, p. 66).

49 Encyclopédie méthodique, Jurisprudence (1784, t. 4, p. 693). Précisons, qu'à la suite de la disparition des galères, au milieu du XVIII ${ }^{e}$ en France, on envoie les condamnés aux galères dans les bagnes nouvellement construits.

50 Vigié (1988, p. 421).
} 
condamnés. La modération des peines invite à ne punir par les galères que les crimes les plus graves.

Autre grand obstacle à la correction et à la réinsertion, l'infamie qui entoure la peine des galères doit subir des modifications. Impossible en effet d'imaginer un forçat libéré, portant sur son corps les stigmates de son passé délinquant - la marque «GAL» derrière l'épaule -, retrouver une place dans la société sans susciter la suspicion. L'infamie n'est toutefois pas totalement rejetée. Deux impératifs punitifs s'entrechoquent ici, raison sans doute des divergences entre les réformateurs: l'efficacité de la peine et la correction du criminel ${ }^{51}$. L'infamie impose une souffrance morale; combinant des effets punitif et préventif sur le condamné et sur le reste de la société, elle contribue à l'efficacité pénale. Mais, à l'inverse, elle stigmatise les forçats et rend difficile toute réinsertion sociale. Deux aménagements au régime d'infamie sont envisagés. De même que la peine ne doit concerner que le coupable, l'infamie visera uniquement le condamné. Des principes comme l'exhérédation sont à abolir. De plus, l'infamie ne doit pas se prolonger au-delà de la peine, sinon elle devient un obstacle à la réinsertion sociale. Beccaria prévoit à cette fin la suppression de la flétrissure imposée aux forçats. D'autres, comme Brissot de Warville, estiment que seuls les condamnés à temps doivent être épargnés par la flétrissure puisqu'ils sont amenés à réintégrer le monde social, au contraire des condamnés à perpétuitét ${ }^{52}$. Ainsi se dessine l'idée d'un usage plus parcimonieux de l'infamie pour en conserver l'efficacité punitive tout en laissant au condamné la possibilité de se réinsérer socialement au terme de sa peine.

La peine des galères gagne également sa place dans les esprits des réformateurs par le substitut avantageux qu'on lui voit à la peine de mort. C'est au nom de l'efficacité pénale et de la prévention que Beccaria, le premier, avance cette proposition ${ }^{53}$. Il part du constat que la sévérité des peines n'est pas propre à prévenir le crime. La peine capitale pose le double problème d'être un spectacle cruel qui endurcit plutôt qu'il ne corrige et de n'avoir un effet que limité dans le temps. Assertion quelque peu paradoxale puisque, si l'effet d'une exécution capitale est d'habituer les spectateurs à l'atrocité, effet néfaste donc, on ne peut regretter qu'il soit d'une durée limitée. Mais Beccaria cherche surtout à pointer la force corrective et préventive des peines fondées sur la durée. La sévérité judiciaire doit se mesurer moins à l'intensité qu'à la durée de la peine. À ce titre, les «travaux forcés à perpétuité» constituent une ressource plus efficace que la peine capitale: la souffrance morale pour le condamné transformé en «bête de somme» est constante et son exposition publique en fait un exemple permanent aux yeux de tous. Dans ce moment charnière, il semble que la nature hybride de la peine des galères lui permette de conserver une place au sein des systèmes élaborés par les réformateurs : convertie en travaux publics, dépouillée d'excès de souffrance, devenue corrective pour le condamné et utile à la société, elle trouve donc une seconde jeunesse au sein de la République des lettres.

Les juges genevois mobilisent-ils ces conceptions nouvelles de la peine des galères pour la réintégrer soudainement dans leur arsenal pénal? Il est difficile - et méthodologiquement problématique - de voir un rapport transparent entre discours

51 Cicchini (2001, pp. 83-84) remarque cet antagonisme à propos de l'infamie dans son analyse de la répression de la désertion.

52 Vigié (1988, p. 425).

53 Beccaria (1764, pp. 48-54). 
et pratiques. Toutefois, certains éléments tendent à montrer des nouveautés dans les motivations et les usages de la peine des galères à Genève à la fin du XVIII ${ }^{e}$ siècle. Dans leurs réquisitoires, les procureurs généraux utilisent parfois l'argument de la clémence pour appuyer leur proposition de condamnation aux galères ${ }^{54}$. Mais c'est l'argument sécuritaire qui est sans doute le plus significatif. La société, dont l'ordre a été menacé par un délinquant, doit être protégée de toute nouvelle atteinte. Neutraliser la «dangerosité» sociale d'un individu devient une préoccupation majeure. Justifiée sur ces bases, une condamnation aux galères tombe en 1770. Elle se solde par un échec: faute de trouver un accord avec le royaume de PiémontSardaigne, les autorités se voient dans l'obligation de commuer la peine des trois hommes qu'elles viennent de condamner ${ }^{55}$. L'argument de l'impératif sécuritaire est repris par le procureur général Barthélemy Galiffe (1736-1816) en 1773 pour motiver une peine de galères ${ }^{56}$. Les juges ne le suivent pas. N'en démordant pas, celui-ci profite d'un autre procès pour lancer une nouvelle salve.

[...] j'estime que Pujol doit être condamné à une peine extraordinaire et je ne pense pas que la circonstance de l'arme dont il a été trouvé muni soit assez aggravante pour changer la peine extraordinaire en une peine capitale. C'est dans ce moment, Magnifiques et très honorés Seigneurs, qu'appellé à préjuger la peine à laquelle le prévenu doit être condamné, connaissant les inconvénients d'une prison perpétuelle et réfléchissant sur le péril qu'il y a de rendre à la société un homme aussi dangereux, je crois devoir prier Vos Seigneuries d'observer l'avantage manifeste qui résulterait pour la société en général d'un concordat avec la France, qui permettrait aux juges de condamner le prévenu et ceux qui dans la suite pourraient commettre des délits de même genre aux galères perpétuelles ${ }^{57}$.

L'appareil pénal manque de solutions pour répondre à l'impératif sécuritaire, selon Galiffe. La peine capitale, dont la sévérité est jugée extrême, est appliquée avec plus de parcimonie dans la deuxième moitié du XVIII ${ }^{\mathrm{e}}$ siècle ${ }^{58}$. Certains cas se révèlent graves sans toutefois nécessiter la mise à mort. La clémence ou l'absence de «preuve complète» imposent une peine qui soit plus modérée tout en garantissant la tranquillité de la société. Or, pour retrancher les individus dangereux du corps social, les juges ne disposent comme autres solutions que de la prison et du bannissement. La première a le défaut de coûter très cher et n'assure pas une sécurité suffisante, vu la fréquence des évasions: la peine d'emprisonnement, même si son usage a tendance à augmenter à la fin du siècle ${ }^{59}$, n'est pas encore une réponse pénale jugée viable. Quant au bannissement, il est d'une inefficacité manifeste: les cas de ruptures de ban sont innombrables. Répondant à l'objectif sécuritaire, la peine des galères apparaît, dans le dernier tiers du XVIII ${ }^{e}$ siècle, comme une solution qui vient combler un manque. Et les conditions d'existence d'une telle peine reposent, selon Galiffe, sur la conclusion d'un accord qu'il appelle de ses vœux avec une puissance voisine. Cette solution, Genève n'est pas le seul État à l'envisager. Les autorités de

\footnotetext{
$\overline{54}$ AEG RC 271, 22 janvier 1770, pp. 34-35.

55 AEG RC 271, 8 décembre 1770, p. 710.

56 AEG PC 12389, 1772-1773. Sur l'argument sécuritaire, voir aussi AEG PC 11942, 1769-1770; AEG PC 12512, 1773; AEG PC 13633, 1781.

57 AEG PC 12512, 1773, «Conclusions ».

58 Porret (1995, p. 384).

59 Roth (1981, p. 61).
} 
Neuchâtel sondent à la même époque la cour de Turin pour savoir si elle serait prête à accueillir des criminels sur ses galères. La réponse est sèche: les galères n'existent plus et l'on ne souhaite de toute façon pas s'occuper de criminels étrangers ${ }^{60}$. Avec la France, la République de Genève trouvera un interlocuteur mieux disposé quelques années plus tard.

Hormis l'objectif de protéger le corps social, on peut difficilement voir chez les praticiens genevois de la justice des références explicites aux principes énoncés par les réformateurs du droit pénal. Il faut dire que les modalités d'exécution de la peine des galères concernent peu les magistrats genevois : les galériens échappent très rapidement à leur autorité. De nouvelles inflexions sont pourtant données à la peine des galères dans les juridictions européennes. Au cours du XVIII ${ }^{\mathrm{e}}$ siècle s'est opérée une lente transformation qui a fait des galériens non plus des hommes de mer, mais des forçats de la terre ferme. La suppression massive des corps de galères au milieu du siècle conduit les autorités à trouver de nouvelles affectations à leurs condamnés. Selon une logique utilitariste, les ports, les arsenaux maritimes, les villes où doivent être engagés de gros travaux, accueillent des galériens devenus forçats. L'afflux de cette main-d'œuvre pénale permet des économies. À Naples, on imagine même l'envoi de galériens au Pérou pour travailler dans les mines ${ }^{61}$. La France se contente de les envoyer dans les bagnes qu'elle a construits à Brest, Toulon, Rochefort ou Lorient. Dans les années 1760-1770 se produit en France un tournant qui vise à exploiter plus avantageusement les capacités professionnelles des forçats ${ }^{62}$. Plutôt que de les traiter comme une main-d'œuvre non qualifiée, on porte une attention particulière à leurs compétences pour les orienter vers des chantiers ou des ateliers où ils pratiqueront leur métier. Pour lutter contre l'école du crime qu'est le bagne, Louis XVI et son administration envisagent en 1783 une réforme: ils souhaitent séparer les forçats en différentes catégories, dissociant notamment les condamnés à vie et les condamnés à temps, et éviter ainsi toute «contamination» des petits délinquants par les grands criminels. Le résultat n'est cependant pas concluant ${ }^{63}$.

Les évolutions dans la pratique des condamnations aux galères à Genève, si elles n'ont rien de spectaculaire, traduisent tout de même une tendance : ce type de pénalité répond désormais avant tout aux besoins de la politique pénale de la République. Stabilisée par l'accord de 1782, utile à la machine judiciaire genevoise, la peine des galères n'a plus l'éclat diplomatique qu'elle avait à l'époque de Louis XIV. La délégation genevoise formée à l'occasion de la remise d'un galérien n'est plus menée que par un capitaine aide-major ou un adjudant de la garnison. Banalisée, mais toujours utilisée parcimonieusement, la peine des galères vise désormais tout autant les Genevois que les étrangers. Il s'agit soit de meurtriers et de voleurs récidivistes - crimes très qualifiés -, soit de déserteurs. En effet, l'ordonnance de Louis XVI datant de décembre 1775, qui écarte la peine de mort pour les déserteurs au profit de la peine des galères, fait office de jurisprudence à Genève durant quelques années. La justice militaire de la République prononce une dizaine de condamnations aux galères entre 1783-1784. Mais les mutations juridiques dans la monarchie française

\footnotetext{
$\overline{60}$ Henry (1984, p. 423).

${ }_{61}$ Angiolini (2006, p. 114).

62 Petit (1991, p. 185).

63 Joannic-Seta (2000, pp. 225-228).
} 
incitent les juges militaires genevois à adopter rapidement une nouvelle tactique punitive pour le délit de désertion ${ }^{64}$.

En définitive, la résurgence de la peine des galères à Genève à la fin du siècle des Lumières semble dépendre d'un contexte pénal en pleine mutation: le recul de la peine capitale, que le procureur général Tronchin (1710-1793) imagine remplacée par une peine de «travaux publics», et la lente montée de l'incarcération laissent ouverte une fenêtre temporelle pour la peine des galères, dont l'efficacité est vantée. En effet, la prison de Genève, bien que louée par le voyageur John Howard de passage en 1781, ne paraît pas encore en état de pouvoir constituer une solution à la répression pénale. Le réformiste genevois Julien Dentand (1736-1817) rêve de mieux :

Rien ne me paraîtrait plus propre à rebuter le voleur, que l'aspect continuel d'une maison de force destinée à tous ceux qui oseraient porter une main criminelle sur le bien d'autrui; où l'on serait détenu pour un terme proportionnel à la valeur $\mathrm{du}$ vol qu'on aurait commis; où l'on se verrait contraint d'acquérir cette valeur par son industrie; dont on ne pourrait point se flatter de s'évader facilement, et dont les prisonniers seraient, en cas de besoin, attachés à des travaux qui en exposeraient quelques-uns aux regards du public. Je m'étonne qu'un plan aussi simple, aussi sûrement efficace, qui rendrait superflue une multitude de lois, qui préviendrait une quantité prodigieuse de jugements arbitraires, n'ait encore été admis chez aucun peuple policé, dans un siècle surtout qui se pique de philosophie ${ }^{65}$.

\section{CONCLUSION: \\ LA FRAGILE CONSTRUCTION DE NORMES DE COOPÉRATION JUDICIAIRE}

La peine des galères à Genève, née conjoncturellement, est très rapidement intégrée à la machine judiciaire de la République. Sur la longue durée, le cas genevois illustre bien le processus d'autonomisation de la peine des galères à l'époque moderne. Libérée de contingences militaires, elle ne sert plus, en France, en Espagne et en Italie, à renflouer les bancs des galères dès le milieu du XVIII ${ }^{e}$ siècle; elle est le seul résultat d'une politique judiciaire. De la même manière, la résurgence de cette peine à Genève à la fin du siècle, alors que le voisin français n'est plus avide en hommes de chiourme, s'explique par l'intérêt judiciaire qu'on y trouve. La ductilité de la peine des galères lui permet de traverser le temps et de s'adapter aux réformes pénales en chantier. À Genève, certes, le nombre de condamnations n'est pas très important, mais cette parcimonie s'explique en bonne partie par les difficultés qu'engendre une externalisation de l'exécution de la peine. Les juges genevois doivent composer avec la volonté - ou l'absence de volonté - française. De plus, pour ne pas risquer de refus, ils se focalisent sur les bons éléments de la population criminelle. Ces difficultés sont compensées par les avantages retirés: une reconnaissance diplomatique marquée, une grande efficacité pénale et des frais judiciaires limités. Plus encore, la dimension spatiale qu'engendrent les remises de galériens permet à l'État de renforcer symboliquement la définition de son territoire souverain.

\footnotetext{
64 Cicchini (2001, pp. 80-82).

65 Dentand (1785, t. 1, pp. 140-141).
} 
Faut-il voir dans la coopération judiciaire autour des galériens les débuts d'un ordre judiciaire qui dépasserait le cadre des États? La réponse est certainement non: la coopération entre Genève et la France, ponctuelle et pragmatique, n'a pas pour vocation à construire un espace judiciaire et juridique nouveau. Elle est fragile, car répondant avant tout à des besoins conjoncturels. Cette fragilité découle d'une tension qui apparaît clairement à la fin du XVIII ${ }^{e}$ siècle. Dans la négociation de l'accord de 1782, le comte de Vergennes (1719-1787), ministre français des Affaires étrangères, souligne l'apport d'une coopération judiciaire avec Genève pour la sécurité régionale. Cette coopération peut voir le jour au nom du principe universel $\mathrm{du}$ «repos de l'humanité»:

J'ai considéré, Messieurs, que la position de Genève vous rendant pour ainsi dire gardiens de la tranquillité de plusieurs États limitrophes et en particulier d'une frontière assez isolée du royaume, il n'était pas indifférent que vous eussiez un moyen de soustraire à la société les criminels de tous pays qui sans avoir mérité la mort ne doivent pas recouvrer une liberté dont il n'est que trop probable qu'ils abuseront. Le soin de les garder devenant une charge trop pesante pour vous, $\mathrm{Sa}$ Majesté a jugé devoir concourir au repos de l'humanité ${ }^{66}$.

L'impératif de sécurité à l'échelle interétatique, dont il ne faut pas omettre ici le caractère rhétorique, va se heurter, au début de la Révolution, à la barrière nouvelle de l'État-nation. L'exécution des peines n'est que la basse besogne du droit régalien de rendre la justice. Un État n'a donc pas à s'en charger pour un autre, affirme l'Abbé Grégoire (1750-1831):

Nous ne devons pas souiller notre liberté par l'esclavage de ceux dont nous devons ignorer les délits. Il répugne à la majesté de la nation et à son roi que nous soyons les geôliers et les exécuteurs des jugements rendus par des puissances étrangères pour des fautes commises hors de la domination française ${ }^{67}$.

Cette conception met fin à la pratique des condamnations aux galères à Genève. Un décret adopté le 20 mai 1790 par l'Assemblée nationale accorde la liberté à deux galériens suisses. C'est le signal d'un renoncement de la part de la France à accueillir des hommes condamnés par des juridictions étrangères au sein de ses bagnes. Genève, sans réceptacle pour ses éventuels galériens, cesse de condamner aux galères. Toutefois, cette pratique a sans doute laissé des traces. Par la délégation punitive qu'elle implique, la peine des galères a engagé la République à un dialogue diplomatique avec le royaume de France voisin. Parfois efficace, parfois laborieuse, la coopération judiciaire n'a pas de base fixe. L'avancée procurée par l'accord de 1782 est l'un des rares éléments stabilisateurs d'une pratique qui se faisait jusque-là au coup par coup. Il n'en reste pas moins que la peine des galères façonne des pratiques et des savoirs qui normalisent quelque peu la coopération judiciaire transfrontalière et forgent ainsi un langage commun contribuant à la lente construction de l'État administratif.

Robin Majeur

22 rue du Grand-Bureau

1227 Genève, Suisse robinmajeur@hotmail.com

\footnotetext{
66 AEG RC 279, 13 décembre 1778, «Lettre de Vergennes », pp. 604-605.

67 Le Courrier de Paris du 24 mai 1790. Cité dans Dumas (2005, p. 80).
} 


\section{BIBLIOGRAPHIE}

Angiolini, F., La pena della galera nella Toscana moderna (1542-1750), in Antonielli, L. (dir.), Carceri, carcerieri, carcerati. Dall'antico regime all'Ottocento, Soveria Mannelli, Rubbettino, 2006, pp. 79-115.

Aymard, M., Chiourmes et galères dans la Méditerranée du XVIe siècle, Histoire économique du monde méditerranéen 1450-1650. Mélanges en l’honneur de Fernand Braudel, Toulouse, Privat, 1973, pp. 49-64.

Bamford, P.W., Fighting ships and prisons: the Mediterranean galleys of France in the age of Louis XIV, Minneapolis, University of Minnesota Press, 1973.

Beccaria, C., Des délits et des peines, Genève, Droz, 1965.

Bourdieu, P., Sur l'État, cours au collège de France (1989-1992), Paris, Seuil, 2012.

Brandli, F., Une résidence en République: le résident de France à Genève et son rôle face aux troubles politiques de 1734 à 1768, Genève, Société d'histoire et d'archéologie de Genève, 2006.

Brandli, F., Le nain et le géant. La République de Genève et la France au XVIII siècle: cultures politiques et diplomatie, Rennes, Presses Universitaires de Rennes, 2012.

Brissot de Warville, J.-P., Les moyens d'adoucir la rigueur des lois pénales en France [...], Châlons-sur-Marne, 1781.

Carpanetto, D., Confini, sovranità politica e questioni religiose nel trattato sabaudo-ginevrino del 1754, in Raviola, B. A. (dir.), Lo spazio sabaudo: intersezioni, frontiere e confini in età moderna, Milan, F. Angeli, 2007.

Cicchini, M., Sa majesté voulant pourvoir d'une manière digne de sa sagesse et de son humanité à la punition des déserteurs..., Crime, Histoire \& Sociétés / Crime, History and Societies, 2001, 5, 1, pp. 75-91.

Dentand, J., Essai de jurisprudence criminelle, 2 vol., Lausanne, 1785.

Dufriche de Valazé, Ch.-E., Lois pénales, Paris, 1784.

Dumas, B., Les Suisses aux galères de France 1601-1793, Yens sur Morges, Cabedita, 2005.

Encyclopédie ou dictionnaire raisonné des sciences, des arts et des métiers [...], Paris, 1757, t. 7, «Galérien».

Encyclopédie méthodique [...], Jurisprudence, t. 4, Paris, Liège, 1784, «Galère» et «Addition au mot galère ».

Foucault, M., Surveiller et punir. Naissance de la prison, Paris, Gallimard, 1975.

Grandjean, M., Genève au secours des galériens pour la foi, 1685-1720, mémoire de licence, Genève, 1981.

Henry, Ph., Crime, justice et société dans la Principauté de Neuchâtel au XVIII siècle : (17071806), Neuchâtel, La Baconnière, 1984.

Joannic-Seta, F., Le Bagne de Brest, 1749-1799: L'émergence d'une institution carcérale au siècle des Lumières, Rennes, Presses Universitaires de Rennes, 2000.

Lo Basso, L., Condannati alla galera nell'Italia dell'età moderna: gli esempi di Venezia e Genova, in Antonielli, L. (dir.), Carceri, carcerieri, carcerati. Dall'antico regime all'Ottocento, Soveria Mannelli, Rubbettino, 2006, pp. 117-144.

Muyart de Vouglans, P.-F., Les Lois criminelles de France dans leur ordre naturel, Paris, 1780.

Pastoret, E., Des lois pénales, Paris, t. 1, 1770.

Pelizaeus, L., Räumliche Bezugsebenen: Grenzüberschreitende Strafverfolgung im Oberrheinischen Kreis und der Steiermark bei der Verschickung auf die Galeeren im 18. Jahrhundert, in Wüst, W., Müller, M. (Hrsg.), Reichskreise und Regionen im frühmodernen Europa - Horizonte und Grenzen im spatial turn, Francfort sur Main, Peter Lang, 2011, pp. 443-463. 
Petit, J.-G., Histoire des galères, bagnes et prisons, XIII ${ }^{e}$-XX $X^{e}$ siècles, Toulouse, Privat, 1991.

Pike, R., Penal Servitude in Early Modern Spain: the Galleys, The Journal of European Economic History, 1982, 11, pp. 197-217.

Porret, M., Le Crime et ses circonstances. De l'esprit de l'arbitraire au siècle des Lumières selon les réquisitoires des procureurs généraux de Genève, Genève, Droz, 1995.

Porret, M., Beccaria: le droit de punir, Paris, Michalon, 2003.

Rappaport, S., La chaîne des forçats : 1792-1836, Paris, Aubier, 2006.

Rizzo, S., «Un petit État désire de se bien limiter avec ses voisins, surtout quand ce sont de grands princes...»: contexte et acteurs du traité de limites de Paris de 1749: les travaux d'approche genevois (1719-1725), mémoire de licence, Faculté des lettres, Université de Genève, 2003.

Roth, R., Pratiques pénitentiaires et théorie sociale. L'exemple de la prison de Genève (18251862), Genève, Droz, 1981.

Schlosser, H., Tre secoli di criminali bavaresi sulle galere veneziane (secoli XVI-XVIII), Quaderni del Centro Tedesco di Studi Veneziani, 1984, 28, pp. 3-37.

Serpillon, F., Code criminel ou commentaire sur l'Ordonnance de 1670, Lyon, t. 4, 1777.

Viaro, A., I forzati sulle galere veneziane (1760-1797), Studi veneziani, 1978, 2, pp. 225-247.

Vigié, M., Le Bagne des philosophes, Revue d'histoire moderne et contemporaine, 1988, 35, pp. 409-433.

Wüst, W., Müller, M. (dir.), Reichskreise und Regionen im frühmodernen Europa - Horizonte und Grenzen im spatial turn, Francfort sur Main, Peter Lang, 2011.

Zysberg, A., Les Galériens: vies et destins de 60000 forçats sur les galères de France 16801748, Paris, Seuil, 1987. 